\title{
Fracture incidence rates in Norwegian children, The Tromsø Study, Fit Futures.
}

Tore Christoffersen, MSc ${ }^{1,2}$, Luai A Ahmed, $\mathrm{PhD}^{1,3}$, Anne Winther, $\mathrm{PhD}^{1,4}$, Ole Andreas Nilsen MSc ${ }^{1}$, Anne-Sofie Furberg, $\mathrm{PhD}^{5}$, Guri Grimnes, $\mathrm{PhD}^{6,7}$, Elaine Dennison, $\mathrm{PhD}^{8,9}$, Jacqueline R Center, MB BS, PhD ${ }^{10,12,13}$, John A Eisman, MB BS, PhD ${ }^{10,11,12,13}$, Nina Emaus $\mathrm{PhD}^{1}$

\section{Affiliation}

1. Department of Health and Care Sciences, UIT The Arctic University of Norway, Tromsø, Norway

2. Finnmark Hospital Trust, Alta, Norway

3. Institute of Public Health, College of Medicine and Health Sciences, United Arab Emirates University, Al Ain, UAE

4. Division of Neurosciences, Orthopedics and Rehabilitation Services, University Hospital of North Norway, Norway

5. Department of Community Medicine, UIT the Arctic University of Norway, Tromsø, Norway

6. Division of Internal Medicine, University Hospital of North Norway, Tromsø, Norway

7. Tromsø Endocrine Research Group, Department of Clinical Medicine, UiT The Arctic University of Norway, Tromsø, Norway

8. MRC Lifecourse Epidemiology Unit, Southampton, UK

9. Victoria University, Wellington, New Zealand

10. Bone Biology Division, Garvan Institute of Medical Research, Sydney, Australia

11. School of Medicine Sydney, The University of Notre Dame Australia, Australia

12. St Vincent`s Clinical School, UNSW, Sydney, Australia

13. Department of Endocrinology, St Vincent's Hospital, Sydney, Australia

Corresponding author:

Tore Christoffersen,

Department of Health and Care Sciences,

UiT The Arctic University of Norway,

Forskningsparken, Sykehusveien 21

NO-9037 Tromsø

Norway

E-mail: tore.christoffersen@uit.no Telephone: +47900 82718 
Summary: This study describes childhood fracture rates in Norway, a country known for high fracture rates in the adult population. Fracture rates correspond with other reports from Scandinavia, although with a slightly higher proportion in girls. Indications of increased vulnerability during stages of puberty require further exploration.

\section{ABSTRACT}

Introduction: Fractures are common injuries during childhood. Incidence rates and patterns vary, but population-based data are scarce. The aim of this study was to describe the sex-, age- and maturation-specific incidence of fractures in a representative population-based sample from a region in Norway.

Methods: All fractures in the population based convenient cohort Fit Futures, comprising 961 adolescents under 18 years, were recorded retrospectively from the local hospital. Details on individual's age and fracture site were recorded. A radiologist confirmed all fractures.

Results: In the period from birth to cohort scanning, the register recorded 316 fractures in 253 individuals. Fractures were more common in boys (35 \%) than in girls (31\%). The overall annual fracture incidence was 204 per 10000 persons-year under the age of 18 and 205 under the age of 16. The majority of fractures involved the upper extremities and the most common site of fracture was the forearm with $24 \%$ of the fractures followed by phalanges with $23 \%$ of the fractures. Fractures peaked in girls at sexual maturation stage 3. Boys had a peak in stage 2. Timing of subsequent fractures were also consistent with stages of sexual maturation.

Conclusions: The overall incidence of fractures in childhood in Northern Norway corresponds with other reports from Scandinavia, although the proportion of fractures in girls is higher than in other studies. Both sexes seem especially vulnerable at stages related to sexual maturation. Whether this reflects bone vulnerability or other changes related to puberty requires further investigation. 
KEY WORDS: Epidemiology; Fracture; Radiology; Childhood; Puberty

\section{Introduction}

Childhood fractures are common, comprising $10 \%$ to $25 \%$ of all pediatric injuries [1-4]. Approximately one in three of every girl and boy will suffer a fracture between birth and adulthood [1, 5-7]. Previous studies have reported a remarkable similarity in fracture sites [7$10]$ and trauma mechanisms $[8,10,11]$. However, there are differences in incidence rates over time and between countries and regions. A recent Swedish report showed increasing incidence after decades of decreasing trends [8]. This in contrast to a Finnish report describing decreasing incidence and changing patterns over the last two decades [12]. Furthermore, a study from Great Britain suggests differences in rates in different British regions [13].

Norway is among the countries with the highest incidence of hip and forearm fractures in adult and elderly populations [14], and geographical differences within the country has been reported $[15,16]$. While several Scandinavian studies have addressed the childhood fracture epidemiology $[1,8,10,17]$, to our knowledge there are only two reports on fracture patterns in Norway [7, 9], none of these from the region above the arctic circle. Furthermore, recent studies suggest childhood fractures as a risk factor for adult fractures $[18,19]$. Therefore, the incidence of childhood fractures is of public health importance. The purpose of this study was to determine the extent of childhood fractures in Northern Norway and to describe patterns by sex, age, site and sexual-maturation in comparison with previous reports.

\section{Materials and Methods}




\section{Study Participants}

The Tromsø Study is a population-based study initiated in 1974. The primary aim was to address the issue of high mortality because of cardiovascular disease in Northern Norway. The study has gradually expanded to include other diseases. A collaboration between the University Hospital of North Norway (UNN HF), UiT-The Arctic University of Norway and the National Public Health Institute led to the Fit Futures (FF) expansion. In 2010/2011, the study investigators invited all first-year upper-secondary school students in the two municipalities Tromsø and Balsfjord to participate in a cross-sectional study FF1. The Norwegian school system entitle every inhabitant, who has completed primary school, three years of upper-secondary education. Although, not all students continue directly to uppersecondary school, this convenient sample represent the normal population. Troms County is located in the northern part of the Scandinavian Peninsula at a latitude of nearly $70^{\circ} \mathrm{N}$. The largest city in the County, Tromsø, has a population of 72,681 (January 1, 2015) while the neighboring municipality, Balsfjord, has a population of 5,720. The invited cohort included 1,117 participants aged 15-19 years. Of these, 1,038 adolescents attended the survey giving a participation rate of almost 93\%. A total of 961 participants under the age of 18 were included in this study.

The recruitment procedures involved a close collaboration between the schools and the research unit. Students received oral and written information about the study at the schools. Participants 16 years and older signed a declaration at the study site and younger individuals brought written permission from their parents or guardians. Dedicated research technicians performed the examinations in the research unit at UNN HF.

Fracture Assessment 
The UNN HF hospital is a secondary care university hospital, also serving as a primary care center for the residents of Tromsø and the surrounding municipalities. UNN HF is the only hospital in the area of Fit Futures participants, providing easy access to free of charge services for pediatric injuries; the radiology department performs virtually all X-rays for possible fractures. All records from the radiology department were retrospectively reviewed for all participants in the Fit Future cohort under 18 years of age in 2010/2011 (n=961) and any fracture from birth to their examination date at the research unit were recorded. Fractures initially treated outside the region were also recorded, as follow-up took place at UNN HF.

For registration purposes, a fracture register template containing details on date of treatment, localization of fracture and body side was used, similar to protocols used in the Tromsø Study [20]. Among others, the template allowed information on trauma energy and number of fractures if a subsequent fracture was sustained. Inclusion criterion for fractures was a radiological confirmation of fracture as stated by radiologist on duty at the time of treatment. Diagnoses debated in follow-up hospital records or solely stated by clinical findings were excluded. Treatment dates and personal identification numbers were used to make sure that each participant was recorded only once for each injury. In the case of multiple fractures, we recorded every fracture as separate events, with the following exceptions: simultaneous fractures of radius and ulna on one forearm, tibia and fibula on one leg, multiple vertebral, multiple skull, toes or fingers were recorded as one fracture. Furthermore, any re-fracture was recorded as separate fracture if fracture lines were confirmed united before a new event.

\section{Maturation Assessment}

Two variables from the FF1 questionnaire were used to assess pubertal stages at the time of fracture in individual participants. Girls were asked, "If you have started menstruating, how old were you when you had your first menstrual period?” The answers were given on a 
continuous scale and were re-coded into a five-stage scale based on literature describing time from onset of puberty to time of menarche [21-23]. This scale was initiated with stage 1 if the fracture occurred more than 2 years before first menstrual period. Stage 2 included fractures from 1 to 2 years before menarche, followed by yearly steps for stages 3 and 4 . Any fractures one year or more after menarche was included in the final stage 5. Boys were asked, "If you have got or started to get pubic hair, how old were you started to get pubic hair?” Based on the timing of male puberty and pubic hair development [21], stage 1 was set by fractures more than 1 year before the report of pubic hair. Stage 2 included fractures in the year before and after pubic hair onset, followed by yearly increments for stage 3 and 4 . The final stage included fractures more than three years after pubic hair development.

The study was approved by The Norwegian Data Protection Authority (reference number 2009/1282) and by The Regional Committee of Medical and Health Research Ethics (reference number 2011/1702/REKnord).

Age and sex specified incidence rates were calculated by dividing the number of new fractures during the specified time interval by the summed person-years of observation. Fractures incidences are multiplied and given per 10,000 person-year for comparison. Pearson's chi-square test and students t-test were used for any between group comparisons. A p-value of less than 0.05 was considered significant. All analyses were performed in SPSS Statistics (SPSS, Inc., Version 22.0 Chicago, IL USA)

\section{Results}

Incidence of fractures

Amongst 961 participants, 253 children (26\%) had a diagnosis of fracture from birth to the date of attending the Fit Future survey. Altogether 316 fractures were registered (Table 1), 54 children experienced two fractures, eight experienced three, and one participant suffered four 
fractures. No multiple fractures beside the pre-defined exceptions were registered. Fractures were slightly more common in boys (34.7\%) than in girls (30.9\%). The overall annual incidence of fractures were 204 per 10,000 person-years under the age of 18 and 205 for children under the age of 16 years (Figure 1). The overall incidence for girls under 18 years was 192 per 10,000 person-years and correspondingly 217 per 10,000 person-years for boys. There was no sex difference with $31(n=469)$ girls and $32(n=492)$ boys suffering more than one fracture.

\section{Anatomic location}

A majority, 66\%, of the fractures involved upper extremities including clavicle, fingers and metacarpal fractures (Table 1). Another 30\% of the fractures involved lower extremities and $4 \%$ of the fractures involved skull, facial, vertebral and pelvic area. Girls suffered $68 \%$ of fractures in upper extremities, 30\% in lower extremities and $2 \%$ of fractures in central areas. The corresponding numbers for boys were 66\%, 29\% and 5\% respectively (Figure 2).

The most common location was forearm fractures (radius/ulna) with an incidence of 49.1 per 10,000 person-years accounting for one quarter of all fractures (Table 1) and nearly 9 of 10 at the distal part of the forearm. The incidence for girls was 48.9 per 10,000 person-years and 49.4 per 10,000 person-years for boys. Furthermore, fingers accounted for $22.5 \%$ of fractures, with an incidence rate in girls of 37.0 per 10,000 person-years and 54.4 per 10,000 personyears in boys. The most common lower limbs fracture location was toes accounting for 52.6 $\%$ of the fractures in lower extremities, while the leg and predominantly the distal tibia/fibula site had an overall annual incidence of 22.6 per 10,000 person-years.

Seasonal variations

As other studies have elucidated seasonal variations, this was also analyzed. Although not statistical significant, fracture incidence peaked in May (Figure 3) and in the second quarter of 
the year ( $p=0.311)$. Interestingly, the same pattern was observed for both upper and lower limb fractures.

Initial fractures - anatomic locations and timing with sexual maturation.

The annual incidence of initial fractures was 164 per 10,000 person-years under the age of 18 and 173 for children aged 0 to 14 years (Figure 4). The incidence in children under 18 years was 151 per 10,000 person-years for girls and 176 per 10,000 person-years for boys.

Initial fracture incidence varied by pubertal stage (Figure 5A). Fracture incidence in girls peaked at stage 3 in sexual maturation with 291 per 10,000 person-years. Fracture incidence of initial fractures in boys peaked at stage 2 of sexual maturation with 355 per 10,000 personyear.

Timing of initial forearm fractures was slightly earlier than the overall peaks at pubertal stage 3 in girls and consistent with the overall peak at stage 2 in boys (Figure 5B). In girls, timing of distal tibia/fibula fractures was consistent with an overall peak at pubertal stage 3 (Figure 5C)

Timing of subsequent fractures consisted with the overall peak at stage 2 in pubertal status for boys, while girls also had a peak at stage 2 (Figure 6).

\section{Discussion}

This study describes population-based findings of age and sex specific fracture incidence in children under 18 years of age in Northern Norway. There was an incidence rate of 204 per 10,000 person-years with boys suffering slightly more fractures than girls. The most common fracture site in the cohort was the forearm, followed by those of fingers and toes. Sixty-three (6\%) of the participants sustained more than one fracture from birth to the date they attended the Fit Future study. Girls had a peak of fracturing at stage 3 of pubertal status and boys had a 
high fracture incidence at stage 2. To our knowledge, this study is the first with data on pubertal stages, indicating that fracture rates do coincide with sexual maturation.

Accidents are a common cause of morbidity in childhood and adolescent, and fractures following accidents are suggested to contribute between $10 \%$ and $25 \%$ of all injuries. In the landmark study of Landin [1] the overall annual incidence of fractures was 257 per 10,000 person years among boys and 165 per 10,000 person years in girls. These rates came from the collection of all radiological reports from the city of Malmo, Sweden and reflected fractures in the late nineteen seventies. In the same study, Landin described an increased risk of childhood fractures over the three last decades before their study. However, in their own follow up study a decade later, the annual incidence decreased to a total of 193 per 10,000 for both sexes during the 1980s [10]. This is virtually identical to our comparable age group rates of 197 per 10,000 person years in girls and 211 per 10,000 person years in boys. In contrast, a recent comprehensive study of Mäyränpää et al [12] showed an overall annual incidence of 163 per 10,000 person years for children aged 0-15 years, slightly lower than our 205 per 10,000 person years in the 0-15 interval. The population-based study from Finland also highlighted the decreasing incidence in fractures after an apparent peak around 1980. Our findings could represent a snapshot of the same overall secular trend seen in other Scandinavian countries, but the reasons for any secular change are unknown.

The fracture ratio between male and female in most pediatric studies has been stable over time, and equal $[1,8,12,13]$. However, while the fracture rates were higher in boys (55\%) in our study and that of Brudvik (7) at 59\%, other studies Mäyränpää et al (63\%), Landin (61\%) and Cooper et al (63\%) report greater differences between the sexes [1, 12, 13]. In recent studies on the physical activity levels in our cohort, we have described similarity in patterns of physical activity between sexes $[24,25]$. This equalizing of male:female activity levels 
may explain the slightly lower ratio of male to female fractures, as suggested by Tiderius [10] following the increasing incidents of forearm fractures in girls from 1975-1979 to 1993-1994.

The most common fracture site in the cohort was the forearm. This is consistent with most of the literature $[7,8,10,12,13]$. In our study, almost one of four fractures was the radius/ulna, virtually identical to what Hedstrom found in Northern Sweden [8]. The distal forearm fractures in the recent Finnish study were more frequent at $30.4 \%$ of fractures overall, and more common in the boys with an incidence of 62.7 per 10, 000 person-years [12]. This contrasts with our incidence of 46 per 10, 000 person-years in boys. Interestingly, the girls in our study had an incidence of 42 per 10,000 person-years, higher than in the Finnish study. This again highlights the before mentioned discussion on a similar fracture risk between girls and boys, possibly related to the more similar physical activity patterns.

Some studies have reported a seasonal variation in incidence rates. Childhood fractures reported from Sweden [1] showed high rates of injuries in May, decreasing during summer before another peak in autumn. In addition, a British study had findings in general accordance with this [13], although with a less sharp decline in summer. Our numbers are in accordance with both Landin and Cooper, with increasing incidence rates in May and early autumn, although we observed a third peak in months prior to the start of winter. However, none of these differences were statistically significant.

While most descriptive studies on childhood fractures have evaluated incidence based on chronological age, no other study to our knowledge has described the incidence rate with respect to sexual maturation. Peak growth velocity in females occurs soon after stage 2 [26], which may represent an age range from 8 to 15 years with median 12.2 years of age [27]. Correspondingly, in boys, the growth spurt varies between 12-17 years, with a median value at 13.6 years described in Norwegian boys [27]. Based on stage of sexual maturation, the 
adolescent is at highest risk at the peak of puberty independent of age. This way of illustrating the incidence could have more biological relevance due to the vulnerability of bone following the rapid growth spurt during puberty.

The biological development of especially cortical bone during puberty has been assessed in other early studies [28, 29]. Garn [28] suggested sex-specific development of cortical bone. While the pre-puberty stage is dominated by periosteal expansion and endocortical resorption in both sexes, girls experience endocortical apposition during the pubertal stages. The prolonged periosteal expansion in boys might explain the accumulated high fracture rates early in puberty in our study. However, what Schoenau et al [29] presented as more efficient pubertal bone development in boys, cannot explain the higher fracture rates in boys.

Another possible explanation of the fracture rates is the behavioral characteristics of adolescence. One hallmark of puberty is the increase in gonadal hormones. Although a simple association of adolescent behavioral change and these hormones are not evident, some behavioral properties are common in mammalians [30]. This includes changes in social behavior and an increase in risk-taking/novelty-seeking activities. In our study, combinations of biological, cultural and behavioral factors such as risk seeking activities and social sexdifferences were not studied. However, having identified the relationship of fractures based on pubertal stages, future investigations could focus on these factors.

The strengths of this study are the population-based design including high attendance in both sexes and the full representation of adolescents from both rural and urban regions. Moreover, the likelihood for capturing virtually every fracture in the cohort strengthens the results due to free health care services in public hospitals and the systematic review, reports and journal notes of the radiologists. There are some limitations to the study. Pubertal status could be more comprehensively approached, as the variables in use needs further validation. However, 
this limitation would most probably blur the consistence of relationship more than being erroneous. Although school drops-out are susceptible to present an unhealthy lifestyle, the high attendance rate minimize non-participation bias. The study is prone to information bias, as it relied on the accuracy of those recording the events. Private medical care treat injuries, especially in children within organized sports. Hence, fractures not registered at UNN HF can occur. However, these events are probably few because of the free services at public hospitals and their role as primary health care provider. The cohort was defined at the initial survey, i.e. prior to any evaluation in relation to fractures.

In conclusion, we present a population-based epidemiological study of childhood fractures in a North-Norwegian region. Our findings support the literature of these fractures as a common problem, with one in four children suffering at least one fracture before adulthood. Compared with other studies, our report demonstrates a smaller sex difference, although childhood fractures still are more common among the boys. The most common fracture site was the distal forearm. Distribution between sexes seems to be more balanced than in other studies. In this study, we identified a close relation in timing of fractures and stages of maturation. Although several biological, social and behavioral factors may explain this relationship, the observed relationship between growth spurt and consequential increased bone vulnerability should be further evaluated.

Acknowledgement: We are grateful to the study participants, the staff at the Clinical Research Unit at University Hospital of North Norway (UNN HF) and the Fit Futures administration for conducting the study. We thank Robert Kechter at Finnmark Hospital Trust and The Garvan Insitutue of Medical Research for office and administration contribution. We also thank The Norwegian Osteoporosis Association for supporting paediatric software and the Northern Norway Regional Health Authorities for funding this work. 
Conflict of interest: Tore Christoffersen, Luai A Ahmed, Anne Winther, Ole-Andreas

Nilsen, Anne-Sofie Furberg, Guri Grimnes, Elaine Dennison, Jacqueline R Center, John A

Eisman and Nina Emaus have no conflict of interest to disclose.

Authors' contribution: Study design: TC, AW, LAA and NE. Study conduct: A-SF, GG and

NE. Data collection: TC, A-SF, GG, NE, OAN and AW. Data analysis: TC, JAE, JRC, LAA, and NE. Data interpretation: TC, JAE, JRC, LAA and NE. Drafting manuscript: TC, JAE, JRC, LAA and NE. Revising manuscript content: TC, JAE, JRC, AW, LAA, GG, OAN, ED and NE. Approving final version of manuscript: TC, JAE, JRC, AW, LAA, GG, OAN, ED and NE. TC, LAA and NE take responsibility for the integrity of the data analysis.

\section{References}

1. Landin, L.A., Fracture patterns in children. Analysis of 8,682 fractures with special reference to incidence, etiology and secular changes in a Swedish urban population 1950-1979. Acta Orthop Scand Suppl, 1983. 202: p. 1-109.

2. Mattila, V., et al., Occurrence and risk factors of unintentional injuries among 12-to 18-yearold Finns--a survey of 8219 adolescents. Eur J Epidemiol, 2004. 19(5): p. 437-44.

3. Jones, G. and H.M. Cooley, Symptomatic fracture incidence in those under 50 years of age in southern Tasmania. J Paediatr Child Health, 2002. 38(3): p. 278-83.

4. Sibert, J.R., G.B. Maddocks, and B.M. Brown, Childhood accidents--an endemic of epidemic proportion. Arch Dis Child, 1981. 56(3): p. 225-7.

5. Lyons, R.A., et al., Children's fractures: a population based study. Inj Prev, 1999. 5(2): p. 12932.

6. Cheng, J.C., et al., A 10-year study of the changes in the pattern and treatment of 6,493 fractures. J Pediatr Orthop, 1999. 19(3): p. 344-50.

7. Brudvik, C. and L.M. Hove, Childhood fractures in Bergen, Norway: identifying high-risk groups and activities. J Pediatr Orthop, 2003. 23(5): p. 629-34.

8. Hedstrom, E.M., et al., Epidemiology of fractures in children and adolescents. Acta Orthop, 2010. 81(1): p. 148-53.

9. Kopjar, B. and T.M. Wickizer, Fractures among children: incidence and impact on daily activities. Inj Prev, 1998. 4(3): p. 194-7.

10. Tiderius, C.J., L. Landin, and H. Duppe, Decreasing incidence of fractures in children: an epidemiological analysis of 1,673 fractures in Malmo, Sweden, 1993-1994. Acta Orthop Scand, 1999. 70(6): p. 622-6.

11. Rennie, L., et al., The epidemiology of fractures in children. Injury, 2007. 38(8): p. 913-22.

12. Mayranpaa, M.K., O. Makitie, and P.E. Kallio, Decreasing incidence and changing pattern of childhood fractures: A population-based study. J Bone Miner Res, 2010. 25(12): p. 2752-9.

13. Cooper, C., et al., Epidemiology of childhood fractures in Britain: a study using the general practice research database. J Bone Miner Res, 2004. 19(12): p. 1976-81.

14. Kanis, J.A., et al., A systematic review of hip fracture incidence and probability of fracture worldwide. Osteoporos Int, 2012. 23(9): p. 2239-56. 
15. Falch, J.A., et al., Secular increase and geographical differences in hip fracture incidence in Norway. Bone, 1993. 14(4): p. 643-5.

16. Sogaard, A.J., et al., Cohort profile: Norwegian Epidemiologic Osteoporosis Studies (NOREPOS). Scand J Public Health, 2014. 42(8): p. 804-13.

17. Lyons, R.A., et al., Incidence and cause of fractures in European districts. Arch Dis Child, 2000. 82(6): p. 452-5.

18. Amin, S., et al., A distal forearm fracture in childhood is associated with an increased risk for future fragility fractures in adult men, but not women. J Bone Miner Res, 2013. 28(8): p. 1751-9.

19. Holloway, K.L., et al., Prior fracture as a risk factor for future fracture in an Australian cohort. Osteoporos Int, 2015. 26(2): p. 629-35.

20. Ahmed, L.A., et al., Progressively increasing fracture risk with advancing age after initial incident fragility fracture: the Tromso study. J Bone Miner Res, 2013. 28(10): p. 2214-21.

21. Coleman, L. and J. Coleman, The measurement of puberty: a review. J Adolesc, 2002. 25(5): p. 535-50.

22. Duke, P.M., I.F. Litt, and R.T. Gross, Adolescents' self-assessment of sexual maturation. Pediatrics, 1980. 66(6): p. 918-20.

23. Gluckman, P.D. and M.A. Hanson, Changing times: the evolution of puberty. Mol Cell Endocrinol, 2006. 254-255: p. 26-31.

24. Winther, A., et al., The Tromso Study: Fit Futures: a study of Norwegian adolescents' lifestyle and bone health. Arch Osteoporos, 2014. 9: p. 185.

25. Christoffersen, T., et al., Does the frequency and intensity of physical activity in adolescence have an impact on bone? The Tromso Study, Fit Futures. BMC Sports Sci Med Rehabil, 2015. 7: p. 26.

26. Tanner, J.M. and P.S. Davies, Clinical longitudinal standards for height and height velocity for North American children. J Pediatr, 1985. 107(3): p. 317-29.

27. Waaler, P.E., Anthropometric studies in Norwegian children. Acta Paediatr Scand Suppl, 1983. 308: p. 1-41.

28. Garn, S.M., The course of bone gain and the phases of bone loss. Orthop Clin North Am, 1972. 3(3): p. 503-20.

29. Schoenau, E., et al., The development of bone strength at the proximal radius during childhood and adolescence. J Clin Endocrinol Metab, 2001. 86(2): p. 613-8.

30. Spear, L.P., The adolescent brain and age-related behavioral manifestations. Neurosci Biobehav Rev, 2000. 24(4): p. 417-63.

\section{Figure legends:}

Figure 1: Annual incidence of all fractures in children by age in years and sex, The Tromsø Study, Fit Futures.

Figure 2: Distribution of all fractures by anatomic region, The Tromsø Study, Fit Futures.

Figure 3: Seasonal variations in childhood fractures, The Tromsø Study, Fit Futures.

Figure 4: Annual incidence of initial fractures by age in years and sex, The Tromsø Study, Fit Futures. 
Figure 5: Distribution of initial fractures indicated by stages of sexual maturation in A) all sites, B) forearm and C) tibia and fibula, The Tromsø Study, Fit Futures.

Figure 6: Distribution of subsequent fractures by stages of sexual maturation, The Tromsø Study, Fit Futures.

Table 1: Fractures site distribution in the Tromsø Study Fit Future cohort, children under 18 years of age. All fractures.

\begin{tabular}{|c|c|c|c|c|c|c|c|c|}
\hline & & \multicolumn{3}{|c|}{ All Children } & \multicolumn{2}{|c|}{ Girls } & \multicolumn{2}{|c|}{ Boys } \\
\hline Fracture site & $\begin{array}{l}\text { Diagnosis } \\
\text { (ICD-10) }\end{array}$ & $\mathbf{n}$ & $\%$ & Incidence $^{a}$ & $\mathbf{n}$ & Incidence & n & Incidence \\
\hline Radius/Ulna & & 76 & 24.1 & 49.1 & 37 & 48.9 & 39 & 49.4 \\
\hline Proximal & S52.0-S52.1 & 5 & & 3.2 & 3 & 4.0 & 2 & 2.5 \\
\hline Diaphyseal & S52.2-S52.4 & 3 & & 1.9 & 2 & 2.6 & 1 & 1.3 \\
\hline Distal & S52.5-S52.8 & 68 & & 43.9 & 32 & 42.3 & 36 & 45.6 \\
\hline Fingers & S62.5-S62.7 & 71 & 22.5 & 45.9 & 28 & 37.0 & 43 & 54.4 \\
\hline Toes & S92.4-S92.5 & 50 & 15.8 & 32.3 & 21 & 27.8 & 29 & 36.7 \\
\hline Tibia/Fibula & & 35 & 11.1 & 22.6 & 19 & 25.1 & 16 & 20.3 \\
\hline Proximal & S82.1 & 4 & & 2.5 & 3 & 4.0 & 1 & 1.3 \\
\hline Diaphyseal & S82.4 & 3 & & 1.9 & 2 & 2.6 & 1 & 1.3 \\
\hline Distal & S82.2 & 28 & & 18.1 & 14 & 18.5 & 14 & 17.7 \\
\hline Humerus & & 32 & 10.1 & 20.7 & 18 & 23.8 & 14 & 17.7 \\
\hline Proximal & $\mathrm{S} 42.2$ & 6 & & 3.9 & 3 & 4.0 & 3 & 3.8 \\
\hline Diaphyseal & S42.3 & 1 & & 0.6 & 0 & 0 & 1 & 1.3 \\
\hline Distal & S42.4 & 25 & & 16.2 & 15 & 19.8 & 10 & 12.7 \\
\hline Clavicle & S42.0 & 30 & 9.5 & 19.4 & 16 & 21.2 & 14 & 17.7 \\
\hline Skull & S02.0-S02.1 & 7 & 2.2 & 4.5 & 0 & 0 & 7 & 8.9 \\
\hline Femur & S72.0-S72.4 & 6 & 1.9 & 3.8 & 2 & 2.6 & 4 & 5.1 \\
\hline Pelvis & S32.3-S32.7 & 2 & 0.6 & 1.3 & 1 & 1.3 & 1 & 1.3 \\
\hline Wrist bones & S62.0-S62.1 & 2 & 0.6 & 1.3 & 0 & 0 & 2 & 2.5 \\
\hline Face & S02.2-S02.7 & 1 & 0.3 & 0.6 & 0 & 0 & 1 & 1.3 \\
\hline Patella & S82.0 & 1 & 0.3 & 0.6 & 1 & 1.3 & 0 & 0 \\
\hline Ribs & S22.3-S22.4 & 1 & 0.3 & 0.6 & 1 & 1.3 & 0 & 0 \\
\hline Tarsal bones & S92.0-S92.2 & 1 & 0.3 & 0.6 & 0 & 0 & 1 & 1.3 \\
\hline Vertebrae & S32.0,S32.7 & 1 & 0.3 & 0.6 & 1 & 1.3 & 0 & 0 \\
\hline Total & & 316 & 100 & 204 & 145 & 191.7 & 171 & 216.5 \\
\hline
\end{tabular}

${ }^{\mathrm{a}}$ Incidence per 10000 persons-year. 


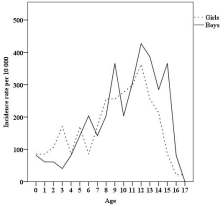




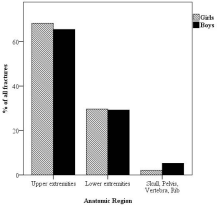




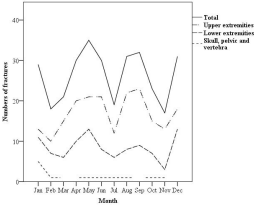




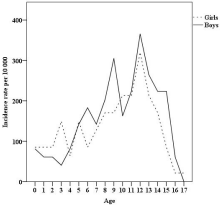




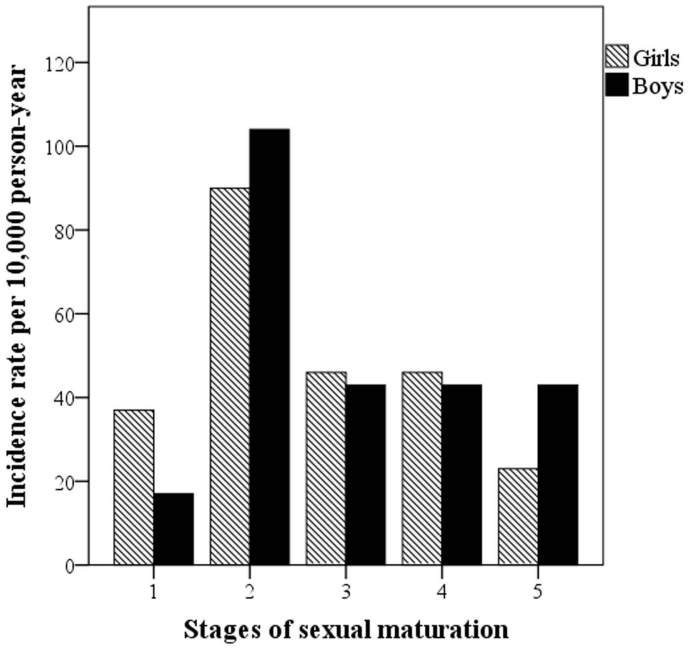

